\title{
Zur Rehabilitierung des Subjektiven
}

\section{Thomas Schweizer}

Dr. med., Facharzt für Allgemeine Innere Medizin, Mitglied FMH

"Wenn ihr's nicht fühlt, ihr werdet's nicht erjagen.» (Goethe, Faust I)

In der Medizin wird die Subjektivität widersprüchlich bewertet. In Bezug auf die Patientinnen und Patienten ist sie die nach wie vor erste Informationsquelle. Sie gibt Einblick in das Krankheitserleben, ist oft subtiler Fingerzeig auf die Ursachen des Leidens und sagt viel aus über Bewältigungsstrategien. In Bezug auf uns Ärztinnen und Ärzte aber steht sie unter Verdacht. Obwohl unabdingbar für Empathie und Intuition, hält man sie für eine gefährliche Quelle verzerrter Wahrnehmungen, die von Ängsten und Hoffnungen statt von der Realität gelenkt sein können. Health Professionals sind dazu aufgerufen, die eigene Subjektivität für das Verstehen der Menschen einzusetzen, das Erfahrene aber ins Prokrustesbett wissenschaftlicher Erkenntnisse zu legen. Es lohnt sich deshalb, grundsätzlicher über das Wesen der Subjektivität nachzudenken und das Spannungsverhältnis Subjektivität-Objektivität sowie, in Analogie dazu, dasjenige zwischen Wahrnehmung und Messung genauer zu betrachten.

\section{Subjektivität: das Unsagbare}

Wer die Subjektivität beschreiben will, steht vor einem Paradoxon. Alle wissen, was sie ist, aber niemand kann sie sagen [1]. Sie verflüchtigt sich im Moment der Definition. Man kann auch den Vergleich zum Traumwandeln anstellen. Im Moment des Erwachens ist die Sicherheit dahin. Subjektivität begrifflich festzumachen heisst, sie

\section{Die Reduktion ist Voraussetzung für alles rationale Erkennen.}

aus der Einheit herauszubrechen, die wir selber sind, aus der Daseinsvergessenheit, in der wir die Welt ungedeutet fühlen, spüren, riechen und sehen, bevor wir überhaupt wissen, dass wir es sind, die sie wahrnehmen. In diesem Beginnen wurzelt unser ursprüngliches, tiefes Gefühl, am Leben zu sein.

Dieses Gefühl schafft eine instinktive Wahrnehmung dessen, was uns am Leben erhält. Eine Art Navigationsfähigkeit zwischen Lust und Unlust, Sicherheit und Gefahr, ein Gespür für unsere existentiellen Bedingungen, für ein Gleichgewicht, das tief in unserem
Sein, in unseren Knochen verankert ist und mit dem Begriff des Bauchgefühls nur oberflächlich umschrieben wird. Der Kampf um Homöostase fundiert unser ganzes Leben. Er ist vielleicht der Grund für die Stabilität des Selbst. Für gewiss und wahr halten wir nur, was wir auch fühlen, was wir erleben. Was einen Zusammenhang hat zu unserem Gleichgewicht. Der Intellektualismus sollte sich das stets vor Augen halten.

\section{Wer die Subjektivität beschreiben will,} steht vor einem Paradoxon.

Unser Anfang im nicht Erinnerten, im Ungenannten, in der Zeit, die noch nicht ins Licht des Rationalen gezerrt ist, fundiert auch unseren primären Wahrnehmungsglauben, aus dem sich noch keine Perspektive löst. Auch er bleibt uns im Kern erhalten als Befähigung zu einer ursprünglichen, unverfälschten Wahrnehmung. Zum naiven Staunen, das unter aller Sozialisierung und aller Lebenserfahrung weiter schlummert.

Was bisher freilich nicht erwähnt wurde, sind die andern. Wir reifen mit Hilfe der andern, der Eltern, der Umgebung, die uns liebt und uns stiftet. Ohne sie gäbe es uns nicht. Diese basale Erfahrung ermöglicht die Intersubjektivität, aus der die Objektivität hervorgeht. Objektivität ist normierende Bezeichnung von Wahrnehmung, strenge Sachlichkeit, Reduktion des subjektiven Erlebens auf das Mess- und Überprüfbare. Sie macht eine Abstraktion notwendig, wörtlich ein Wegziehen von Eigenschaften von den Phänomenen der erlebten Welt, bis das Übrigbleibende mit anderen Interessierten auf einen gemeinsamen Nenner gebracht werden kann. Die Reduktion ist Voraussetzung für alles rationale Erkennen. Objektivität geht aus der Subjektivität hervor und befähigt uns zu einem gemeinsamen, kontrollierten Blick auf die Welt. Man kann den Prozess der Objektivierung auch mit der Sprachbildung vergleichen. Worte erreichen die Phänomene des Lebens ebenfalls nie ganz. In ihnen schwingt ein atmosphärischer Überschuss mit, der auf das Ungesagte im Gesagten verweist. Worte reduzieren.

Dennoch ist Sprache plastisch. Sie verändert sich in ihrer Interaktion mit dem betasteten Gegenstand. Plastisch sind auch Theoriebildung und Methoden der Wissenschaften. Die empirischen Daten sind oft verwirrend, und deren Deutung, die Theoriebildung, bedarf der In- 
tuition und Spontaneität der suchenden Wissenschaftler. Sie müssen in solchen Momenten alles Wissen für einen Moment vergessen und ihrer unvoreingenommenen Fähigkeit des Staunens, der Subjektivität erliegen. Es ist eine Art Sauerstoffzufuhr zu den erarbeiteten Daten. Dieser frische Wind weht freilich meistens nur bei den Fleissigen, denen, die sich in der Empirie abgemüht haben. Aber ohne subjektives Empfinden sind wir zum Theorein, wörtlich zum Schauen, nicht fähig. Das nicht Rationale, das darin enthalten ist, hilft manchmal, den Schleier vor der Natur, den das rein kausal-analytische Denken nicht durchdringen kann, ein wenig anzuheben.

\section{Subjektivität und Objektivität: ein komplementäres Verhältnis}

Subjektivität und Objektivität sind also nicht das simple Gegensatzpaar, für das man sie hält. Sie sind komplementär. Diese Komplementarität ist uns im Alltag vertraut. Wir pendeln immer zwischen der Perspektive der ersten und der dritten Person hin und her, zwischen Erleben und Benennen, Deuten und Prüfen, Wahrnehmen und Messen. Befremdlich ist nur, dass die Objektivität ihre Grenzen und ihre Herkunft vergisst und sich aufspreizt. Die Patientinnen und Patienten spüren das

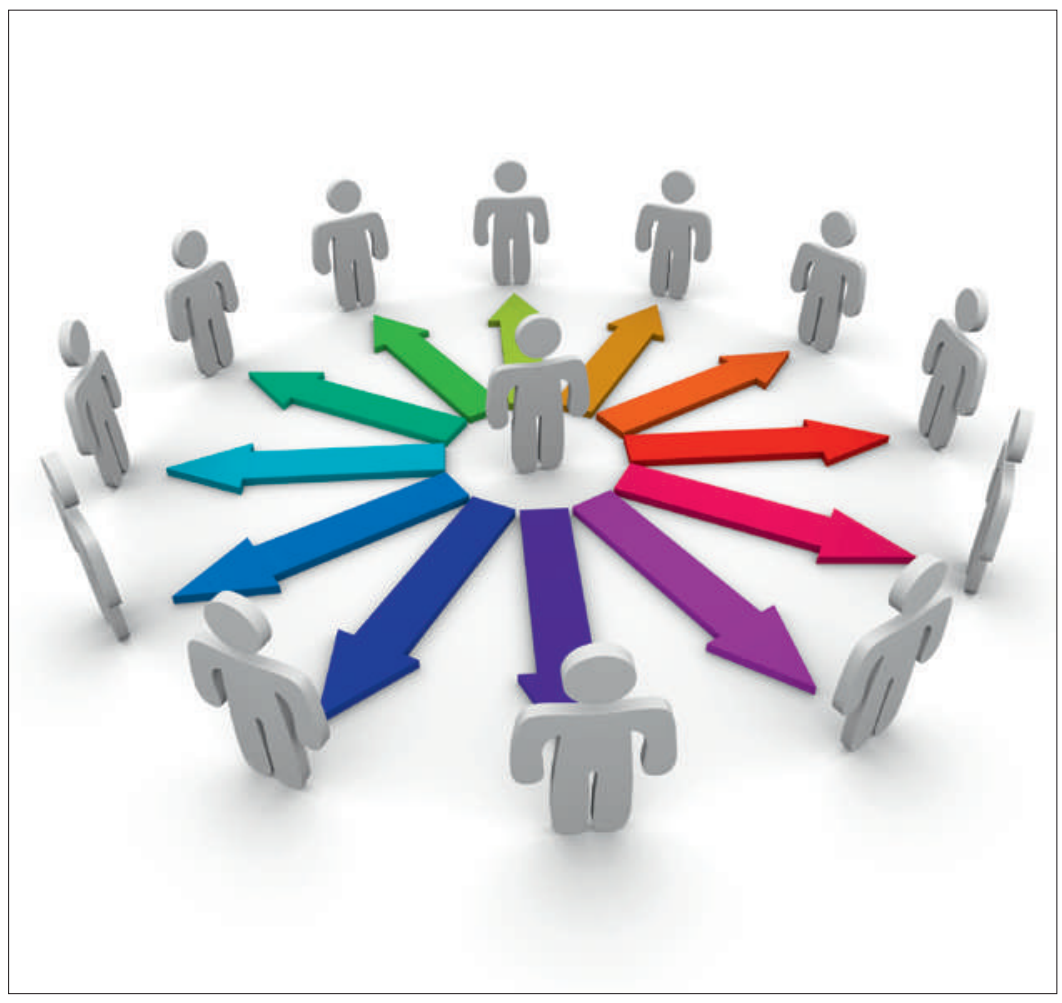

Interessant ist, wie Leute ihr Leiden bei verschiedenen Zuhörern verschieden erzählen. Ein Hausarzt, ein Spezialist, ein Theologe und ein Psychologe erhalten, wie Erfahrungen in entsprechenden Institutionen gezeigt haben, alle nicht die gleichen Auskünfte. Verschieden dürften demnach auch Auskünfte bei alternativ tätigen Kollegen und esoterischen Therapeuten sein. und sind ambivalent. Ungläubig staunen sie darüber, dass die Medizin so viel mehr über sie weiss als sie selbst, dass ihr eigenes Erleben der wissenschaftlichen Prüfung bedarf. Sie wollen zwar den Segen der Technik nicht missen, aber trotzdem von ihrer Subjektivität her verstanden werden. Oft sind wir mit ihrem Doppelanspruch konfrontiert, der zum double bind werden kann: Ihr sollt meinen Körper wissen, aber er ist anders. Dessen rein objektive, physikalistische Erforschung ist in Gefahr, ihn bloss als Körpermodell ohne Subjektivität, als Vexierbild der Naturwissenschaft zu zeigen. $\mathrm{Zu}$ teilen, was eine Einheit ist. [2]

Der Triumph der exakten Naturwissenschaften hat dazu geführt, dass das Subjekt und seine Wahrnehmung an den Rand gedrängt werden, und dass der Machtanspruch der Medizin die Einheit von Gefühl und Verstand, von Sinn und Machbarkeit, von Psyche und Soma aufbricht. Diese Einheit kann nicht durch einen Vorsatz bewahrt oder wiederhergestellt werden, nicht durch eine bio-psycho-soziale Medizin und auch nicht durch die Erklärung, dass der Patient im Zentrum stehe. Sie kann nur wiederentdeckt werden, und zwar im Subjekt selbst. Nur dieses kann die Gedanken auch fühlen, fühlen, was Sinn macht, bis es die Gewissheit hat, was zu tun und was zu lassen sei. Wann es genug ist.

Einheit entsteht in der Wahrnehmung. Wahrnehmen ist wesentlich erleben. Man müsse die Natur «sein», die man verstehen wolle, schrieb Viktor von Weizsäcker, denn nur in der erlebten Interaktion sei das Objekt erfahrbar. «Wir nehmen Dinge notwendig so wahr, wie sie unter Bedingungen erscheinen müssen» [3]. Die Bedingung ist vor allem andern unsere eigene Natur. Die Welt erscheint uns so, wie wir sie fassen können. Wir haben kein Gottesauge, das über ihr schwebt. Wir erleben die Interaktion mit ihr. Diese Interaktion ist konstitutiv für unsere Erkenntnis. Ihr Ziel und Zweck ist Kohärenz. Wir müssen die Welt auf die Reihe kriegen. Auch die Objektivität gibt kein Gottesauge. Wenn wir nach Methoden suchen, um den Phänomenen rational näher zu kommen, so sind auch diese Methoden den Bedingungen der menschlichen Natur unterworfen. Unschärferelation und Quantenphysik haben gezeigt, dass Messen das Gemessene verändert. Die Information kann von der Wirklichkeit nicht getrennt werden. Die Natur interagiert mit uns. Sie ist nicht das Objekt, das in der res extensa aufgespannt werden kann. «Es gibt keinen Punkt in der Einheit der Handlung, an dem mein Selbst aufhört und die Welt beginnt», meint der Philosoph Thomas Fuchs [4].

Die Wahrnehmung, sei sie die genuine oder die rationalisierte, kann als ein Spiel der Natur bezeichnet werden. Die Natur spielt. Es ist kein trügerisches Spiel, vielmehr ein Tanz der Notwendigkeit mit der Möglich- 
keit. Spielend erfahren wir, dass es Wirklichkeit, aber nicht Wahrheit gibt in einer sich wandelnden Welt. Suchend pendeln wir zwischen Erleben und Benennen. Beides kann in stillen Augenblicken verschmelzen. Es sind Augenblicke der Einheit und Selbstvergessenheit,

\section{Wir erkennen die Kompetenz der Patientinnen und Patienten, wenn wir achtsam sind.}

vergleichbar vielleicht dem Moment des Einschlummerns, in dem alles verschwimmt und alles stimmt. Ärztinnen, Patienten, Pflegende, alle Beteiligten können solche Momente erleben. Es ist in ihnen Erkenntnispotential verborgen.

\section{Ärztinnen und Ärzte laufen Gefahr, dem Patienten seinen Körper zu entwenden}

In ihrer reinsten Form ist die menschliche Wahrnehmung absichtslos. In Bezug auf die Patienten kann sie Hinweise geben auf das, was wir genauer betrachten müssen und wissenschaftlich weiter erkunden wollen, und sie ist Hilfe auch bei der Interpretation von Messresultaten. Ansonsten wird das Objektive selbst handlungsanleitend in rein rationalem Sinn. Als Ärztin und Arzt ist man dann in Gefahr, ständig zu handeln, von Machbarkeiten getrieben zu werden und dem Patienten den Körper zu entwenden. Wir spüren den Konflikt täglich, denn wir nehmen immer mehr wahr als unsere
Befunde. Gott sei Dank: Wir "merken» die Leute. Der Konflikt sollte gar keiner sein. Die Wahrnehmung ist wegleitend. Sie ist der viel gelobte ganzheitliche $\mathrm{Zu}$ gang. In ihrer absichtslosen Form ist sie kompetent. Sie wird uns helfen, den wissenschaftlichen Befund zum Nutzen der Leute zu gebrauchen. Auch für sie ist das Vertrauen in die eigene Wahrnehmung entscheidend. Denn in einer therapeutischen Begegnung sollte immer ein Drittes entstehen, das der Arzt oder der Patient alleine nicht hätten erkennen können. Wir merken die Kompetenz der Patientinnen und Patienten, wenn wir achtsam sind. Sie macht ihnen schlussendlich auch den Weg frei aus der Unmündigkeit, in die sie durch die Medizin geraten sind. Eigentlich möchte die Wahrnehmung auch uns Health Professionals befreien und uns das Vertrauen in die eigene Subjektivität zurückgeben. Denn keine künstliche Intelligenz und keine findige, smarte IT-Exploration wird das Subjekt und seine Wahrnehmung je erreichen können.

\section{Literatur}

1 Vogelsang F. Offene Wirklichkeit. Freiburg i. Br.: Karl Alber; 2011.

2 Rappe G. Leib und Subjekt. Bochum/Freiburg: projekt verlag; 2012.

3 Weizsäcker von V. Gesammelte Schriften, Band 4. Frankfurt a. M.: Suhrkamp; 1997.

4 Fuchs T. Lebendiger Geist In: Postphysikalismus. Freiburg i. Br.: 2011; Karl Alber.

Bildnachweis

(c) Iqoncept | Dreamstime.com
Tavelweg 16

CH-3006 Bern

famschweizer[at]bluewin.ch 